\title{
RCSDN: A Distributed Balanced Routing Algorithm with Optimized Cluster Distribution
}

\author{
Arash Ghorbannia Delavar, Javad Artin, and Mohammad Mahdi Tajari
}

\begin{abstract}
In this paper we will present a distributed balanced routing algorithm with optimized cluster distribution. According to the previous clustering algorithms, selection conditions for the cluster head $(\mathrm{CH})$ were either accidentally evaluated or evaluated based on some parameters regardless of the state of a node or its neighbors. In the presented algorithm a type of mechanism was used in which it performs cluster formation in special conditions with regard of the neighborhood principle and local information of a node and its neighbors. In RCSDN a new technique is used with which we were able to apply a selective condition by using a local and distributed threshold detector so that a more optimized method for selecting CHs is created. Finally, with the results of multiple simulations we were able to show that RCSDN, in comparison with the previous Clustering Algorithm has increased the lifetime of sensor network, and reduced the amount of node energy consumption by balancing the use of energy between nodes, therefore resulting to a more suitable distribution of clusters in the sensor network. Hence, this algorithm is more effective compared to the previous algorithms.
\end{abstract}

Index Terms-Clustering, wireless sensor network, energy balancing, energy efficiency, routing, distributed system.

\section{INTRODUCTION}

During the Twenties century, man has used sensors for the means of monitoring his surroundings. As a result of the advances in wireless communication and electronics technologies, wireless sensors are getting smaller, cheaper, and more powerful. The development of these miniaturized wireless sensors enables to use sensor networks for many applications such as military surveillance, environmental monitoring, infrastructure and facility diagnosis, and other commercial applications [1]-[4] .These sensors monitored their environment and transformed the received information into an electrical signal. The signal processing method has a close relationship with the event type and the subject under review. Sensors present in the environment send their data to a data center or base station (BS) for review and further decisions [5]. Despite proper performance and high efficiency levels have a number of limitations in high-scale development. Limitations of the sensors can be grouped in one of the following forms: low energy power levels, and the lack of battery replacement in most cases, limitation of their

Manuscript received April 10, 2012; revised July 13, 2012. This work was supported in part by the Computer Department of Payame Noor Universtiy, PO BOX 19395-3697, Tehran, IRAN.

Arash Ghorbannia Delavar and Javad Artin Delavar are with the Computer Department of Payame Noor Universtiy (e-mail: a_ghorbannia@pnu.ac.ir, javad.artin@gmail.com).

Mohammad Mahdi Tajari Delavar is with the Computer Department of Islamic Azad University (e-mail: mm.tajari@gmail.com). bandwidth and short radio range. Managing a large number of nodes with these limitations can provide many challenges [6]. Energy limitation of the nodes has caused that the reduction of energy consumption in all layers of sensor network design, be considered as one of the main cases. One of the most important layers in this case is the network layer in which the routing process is done. The routing notion in sensor networks is distinguished from other wireless communication networks because of some intrinsic characteristic and has placed more challenges in the path of the design. Some of its intrinsic characteristic includes: lack of a broad IP creation for every node and in result the inability of execution of the many common network routing algorithms, which result to an increase in data traffic in these networks and also limitations in the energy of message transmission, limitations in the power present in each node, and limitations in the calculation potency and the memory on nodes.

Regarding the mentioned cases, a lot of routing methods were created for WSN which can be divided into three groups base on the most common categorization: Data -centric Algorithms, Hierarchical Algorithms and Location Base Algorithms [6]. Data-centric protocols are query-based and depend on the naming of desired data, which helps in eliminating many redundant transmissions. Hierarchical protocols aim at clustering the nodes so that cluster heads can do some aggregation and reduction of data in order to save energy. Location-based protocols utilize the position information to relay the data to the desired regions rather than the whole network [6]. Cluster based methods benefit from a few characteristics: the first characteristic is that they divide the network into several parts and every part is directed by one cluster head. This characteristic causes the cluster based methods to be of a higher scalability level. The second characteristic is that a cluster head receives the data of its nodes and sends it BS after gathering data, which results in substantial reduction in data redundancy. We will also provide a clustering algorithm, which uses a new distributed method, and a local threshold detector to perform clustering. By comparing RCSDN to previous algorithms we will evaluate it.

\section{RELATED WORKS}

One of the most famous clustering algorithms is LEACH [7]. The operation of LEACH is divided into rounds and each round separated into two phases, the set-up phase and the steady-state phase. In the set-up phase, each node decides whether or not to become a cluster head for the current round. This decision is based on the threshold $\mathrm{T}(\mathrm{n})$ given by: 


$$
T(n)= \begin{cases}\frac{p}{1-p *\left(r \bmod \frac{1}{p}\right)} & \text { if } n \in G, \\ 0 & \text { otherwise }\end{cases}
$$

where $\mathrm{p}$ is the predetermined percentage of cluster heads (e.g., $p=0.05), r$ is the current round, and $G$ is the set of nodes that have not been cluster heads in the last $1 / \mathrm{p}$ rounds. Cluster head broadcasts an advertisement message to the rest of the nodes. Depending on the signal strength of the advertisement messages, each node selects the cluster head it will belong to. The cluster head creates a Time Division Multiple Access (TDMA) scheme and assigns each node a time slot. In the steady-state phase, the cluster heads collect data from sensor nodes, aggregate the data and send it to the base station. Since the decision to change the $\mathrm{CH}$ is probabilistic, there is a good chance that a node with very low energy gets selected as a $\mathrm{CH}$. When this node dies, the whole cell becomes dysfunctional. Also, the $\mathrm{CH}$ is assumed to have a long communication range so that the data can reach the base-station from the $\mathrm{CH}$ directly. This is not always a realistic assumption since the $\mathrm{CHs}$ are regular sensors and the base-station is often not directly reachable to all nodes due to signal propagation problems. [8]

LEACH-Centralized [9] uses a centralized clustering algorithm. In setup phase, the base station receives all information about each node regarding their location and energy status. The base station runs local algorithm for the formation of cluster heads and clusters and broadcasts a message that contains the cluster head ID for each node. The steady-state phase of LEACH-C is identical to that of the LEACH protocol. Because the clustering setup is done every round a significant amount of energy is consumed and communication latency is increased.

HEED [10] provides balanced cluster heads and smaller sized clusters. They use two radio transmission power levels; one for intra-cluster communication and the other for inter-cluster communication. HEED does not select cluster head nodes randomly. Sensor nodes that have a high residual energy can become cluster head nodes. But the process of selecting the cluster head uses a complex algorithm which consumes a lot of energy in the network.

Now by using the presented algorithm we can use a new selection conditions for creating clusters which reduces node energy consumption, and balances node energy consumption by the appropriate distribution of cluster head in the network; and create suitable conditions compared to previous methods.

\section{NETWORK MODEL AND ENERGY MODEL}

\section{A. Network Model}

The network model of the RCSDN which is under study contains the following characteristics:

1) All sensor nodes and the base station are stationary after deployment.

2) The basic energy of nodes is different.

3) Sensor nodes do not require GPS-like hardware. So, they are not location aware

\section{B. Energy Model}

Aszd Generally, sensors consume energy when they sense, receive and transmit data [11]. Our energy model for the sensors is based on the first order radio model as used [7]. In this model, the transmitter has power control abilities to dissipate minimal energy to send data to the receiver. In order to achieve an acceptable signal-to-noise-ratio (SNR), the energy consumption of the transmitter is given by:

$$
\begin{cases}E_{T x}(n, d)=n\left(E_{\text {elec }}+\varepsilon_{f s} d^{2}\right) & d<d_{0} \\ E_{T x}(n, d)=n\left(E_{\text {elec }}+\varepsilon_{m p} d^{4}\right) & d \geq d_{0}\end{cases}
$$

where, $\mathrm{n}$ is the number bit of the message and $\mathrm{d}$ is the distance. $\underline{E}_{\text {elec }}$ is the energy dissipated per bit to run the transmitter or the receiver circuit, and ${ }_{f s}, m p$ is the energy dissipated per bit to run the transmit amplifier depending on the distance between the transmitter and receiver. If the distance is less than a threshold $d_{0}$, the free space (FS) model is used; otherwise, the multipath (MP) model is used.

The energy consumption of the receiver is given by:

$$
E_{R x}(n)=n\left(E_{\text {elec }}\right)
$$

\section{The New PRESENTED Algorithm RCSDN}

RCSDN is created based on rounds in which each round contains two phases: (1) set-up and (2) steady-state. In the Set-up Phase, the $\mathrm{CHs}$ are determined and the cluster structures are formed. In Steady-state Phase the nodes send the data they've received from the environment to the corresponding $\mathrm{CH}$ and after gathering data in the cluster head, data will be sent to the BS. In the beginning of each round all of nodes have a normal state.

The Set-up Phase in RCSDN starts by sending a start message via $\mathrm{BS}$ with a specified range to the environment. After a node receives the start message from the BS, it provides a relative estimate of its distance from the BS through the intensity of the received signal. Then it broadcasts a message for its neighboring nodes including ID, the distance to BS, and the level of remaining energy. Nodes bound in the radio range of this message, receive it and set this node as a neighbor node, and register its ID and energy level in their memory; Again they proceed to estimate their distance with the neighboring node by calculating the intensity of the received signal and finally calculate their distance from the BS. This is the done by all nodes in the network and all nodes will acquire the ID, level of energy and distance of all neighboring nodes.

In RCSDN we use a local threshold detector $\left(\mathrm{T}_{\mathrm{D}}\right)$ so that only nodes having selection conditions participate in competition for selecting $\mathrm{CH}$. This threshold detector is locally calculated in each node to prevent the lack-of-candidates problem in some areas because of the central selection. The following qualification function is used to prevent this problem: 


$$
T_{D}\left(S_{i}\right)=\frac{\left(\sum_{k=1}^{n_{\text {deg ree }}\left(S_{i}\right)} E_{r e}\left(S_{k}\right)\right)+E_{r e}\left(S_{i}\right)}{k+1}
$$

A Where $n_{\text {degree }}\left(S_{i}\right)$ is equal to the number of neighbors of the node "i". $E_{r e}\left(S_{k}\right)$ is the remaining energy of the neighbor " $\mathrm{k}$ ". $E_{r e}\left(S_{i}\right)$ is the remaining energy of the node "i". Each node decides to become a candidate or not, based on the following relation:

$$
\begin{gathered}
\text { if }\left(E_{\text {re }}\left(S_{i}\right) \geq T_{D}\right) \\
\operatorname{state}\left(S_{i}\right)=\text { candidate } \\
\text { else } \\
\operatorname{state}\left(S_{i}\right)=\text { normal }
\end{gathered}
$$

After distributing the following start message based on the following algorithm which is implemented in each node, the proper $\mathrm{CHs}$ are selected and clusters are formed. In which $\mathrm{S}_{\mathrm{n}}$ is the collection of neighbor nodes in radio range of each node and is defined as:

$$
S_{n}\left(S_{i}\right)=\left\{S_{j} \mid d\left(S_{i}, S_{j}\right) \leq R\left(S_{i}\right)\right\}
$$

\section{Algorithm $1 \mathrm{CH}$ selection and cluster formation}

1. $\operatorname{state}\left(S_{i}\right)=$ normal

2. calculate $T_{D}\left(S_{i}\right)$

3. if $E_{r e}\left(S_{i}\right) \geq T_{D}\left(S_{i}\right)$

4. $\quad \operatorname{state}\left(S_{i}\right)=$ candidate- $\mathrm{CH}$

5. end if

6. Initialize $\mathrm{T}$

7. While $\left(\left(\operatorname{state}\left(S_{i}\right)=\right.\right.$ candidate-CH) OR $\left(\operatorname{state}\left(S_{i}\right)=\right.$ normal)) AND (timer $<\mathrm{T})$ )

8. if $\left(\left(\operatorname{state}\left(S_{i}\right)=\right.\right.$ candidate-CH) AND $\left.\left(\forall S_{j} \in S_{n}\left(S_{i}\right) \mid E_{r e}\left(S_{i}\right)>E_{r e}\left(S_{j}\right)\right)\right)$

9. $\quad \operatorname{state}\left(S_{i}\right)=\mathrm{CH}$

10. broadcast a $\mathrm{CH}\left(\operatorname{Id}\left(S_{i}\right)\right)$ message

11. break

12. end if

13. if ((received a $\mathrm{CH}\left(\operatorname{Id}\left(S_{j}\right)\right)$ message) AND

$\left.\left(S_{j} \in S_{n}\left(S_{i}\right)\right)\right)$

14.

15.

16.

17.

18.

if $\left(\operatorname{state}\left(S_{i}\right)=\right.$ normal $)$

$\operatorname{state}\left(S_{i}\right)=$ cluster-member

$$
C H\left(S_{i}\right)=S_{j}
$$

break

else if $\left(\operatorname{state}\left(S_{i}\right)=\right.$ candidate- $\left.\mathrm{CH}\right)$

$\operatorname{state}\left(S_{i}\right)=$ cluster-member

$$
C H\left(S_{i}\right)=S_{j}
$$

broadcast a Abort $\left(\operatorname{Id}\left(S_{i}\right)\right)$ message

$$
\begin{aligned}
& \text { break } \\
& \text { end if } \\
& \text { end if }
\end{aligned}
$$

if ((received a Abort $\left(\operatorname{Id}\left(S_{j}\right)\right)$ message) AND

$\left(S_{j} \in S_{n}\left(S_{i}\right)\right)$

26.

27.

$S_{n}\left(S_{i}\right)=S_{n}\left(S_{i}\right)-S_{j}$

28. recalculate $T_{D}\left(S_{i}\right)$

if $E_{r e}\left(S_{i}\right) \geq T_{D}\left(S_{i}\right)$
29.

30.

31.

32. end

33. if $\left(\left(\operatorname{state}\left(S_{i}\right)=\right.\right.$ candidate-CH) $\mathbf{O R}\left(\operatorname{state}\left(S_{i}\right)=\right.$ normal $\left.)\right)$

34. $\operatorname{state}\left(S_{i}\right)=\mathrm{CH}$

35. broadcast a $\mathrm{CH}\left(S_{i} . I d\right)$ message

36. end if

In this Algorithm, each node performs a reversed timer regarding its remaining energy level. If this timer concludes and no message of cluster head formation is received from other nodes or the node has not been set as a $\mathrm{CH}$, it will introduce itself as a $\mathrm{CH}$ and broadcast a $\mathrm{CH}$ formation message including its ID.

If the energy of the candidate node is more than the energy of its neighbors, it will introduce itself as a $\mathrm{CH}$ via message broadcast. When a node receives the $\mathrm{CH}$ message, if it has a normal state, it will immediately joined the chosen $\mathrm{CH}$, and change its state to cluster-member. But if it has the candidate- $\mathrm{CH}$ state, it will perform the following actions:

- Change its state to cluster-member.

- Create an abort message containing its ID and broadcast it to its neighbors

When a node receives an abort message from its neighbors, if the state of the node is set to normal or candidate- $\mathrm{CH}$, it will delete the node which sent the message from its neighbors list and proceed to recalculating the threshold detector. Again it compares its remaining energy to the threshold, and it's possible that a node that used to be in a normal state, to turn to a candidate- $\mathrm{CH}$ state; this process of $\mathrm{CH}$ selection guarantees full network coverage. Remember that if in RCSDN, a node receives multiple $\mathrm{CH}$ messages, it will joins a $\mathrm{CH}$ which is closest to it. After this stage, each node will have one of the two states: $\mathrm{CH}$ or cluster-member. After the clusters are formed, each node sends its data to its corresponding $\mathrm{CH}$, which after receiving and gathering the data of its cluster members, will send them to a BS.

\section{Simulation}

We will analyze the presented algorithm in MATLAB by using this issue: The number of live nodes in the algorithm execution rounds, the number of clusters in each round, the distribution of the number of clusters in different rounds and finally the lifetime of the network, by changing the number of nodes in the presented algorithm.

The parameters used in stimulation, are as following; in which the basic node energy is a random amount between 0.3 and 0.5 ; and the nodes are distributed randomly in a quadrangle square perimeter.

\section{TABLE I: SIMULATION PARAMETERS}

\begin{tabular}{ll}
\hline \hline Parameter & Value \\
\hline Network size & $100 * 100 \mathrm{~m}$ \\
Base station location & $50,50 \mathrm{~m}$ \\
Initial energy for node & rand $[0.3,0.5] \mathrm{J}$ \\
$\mathrm{E}_{\text {elec }}$ & $50 \mathrm{~nJ} / \mathrm{bit}$ \\
$\varepsilon_{\mathrm{fs}}$ & $10 \mathrm{pj} / \mathrm{bit} / \mathrm{m}^{2}$ \\
$\varepsilon_{\mathrm{mp}}$ & $0.0013 \mathrm{pj} / \mathrm{bit}^{\mathrm{m}} \mathrm{m}^{4}$ \\
Data aggregation energy & $5 \mathrm{nj} / \mathrm{bit} / \mathrm{signal}$ \\
$\mathrm{d}_{0}$ & 87 \\
\hline \hline
\end{tabular}




\section{A. The Number of Alive Nodes}

We have compared the presented Algorithm with the LEACH [7] method. Fig. 1 shows the total number of nodes alive through simulation time. The figure suggests that in RCSDN, the nodes have longer lifetime than that in LEACH. RCSDN reduces energy consumption and then prolong network lifetime.

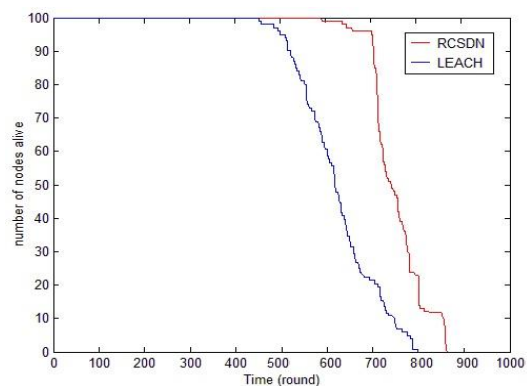

Fig. 1. Total number of alive nodes in the RCSDN and LEACH

\section{B. The Number of Clusters in Different Rounds}

We have compared the number of clusters in different rounds of the network lifetime with LEACH to get reach first "dead". As the figure shows the number of clusters in LEACH method does not have a special balance, and in some rounds this number is very low or very high; but in our presented algorithm the number of clusters in its distribution throughout the network has a good balance, which is because of using local threshold detector, which results in the balance of node energy consumption and increased lifetime of the network.

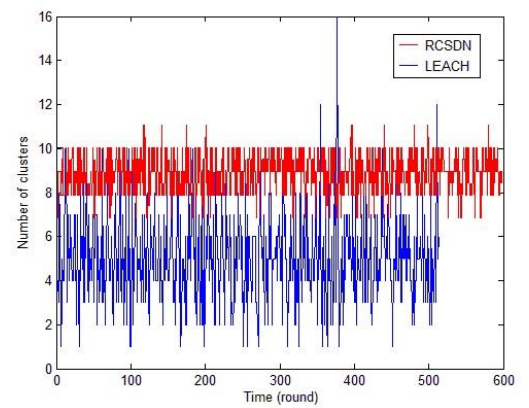

Fig. 2. Total number of clusters in different round

\section{Network Lifetime with Different Number of Nodes}

We performed RCSDN on a network which contained some variable amount of nodes in an environment with a fixed size and compared the results with LEACH and as you see the lifetime of the presented algorithm is more. The cause of this is the appropriate distribution of clusters in the network and the consideration of the local state of the node and its neighbors in cluster formation.

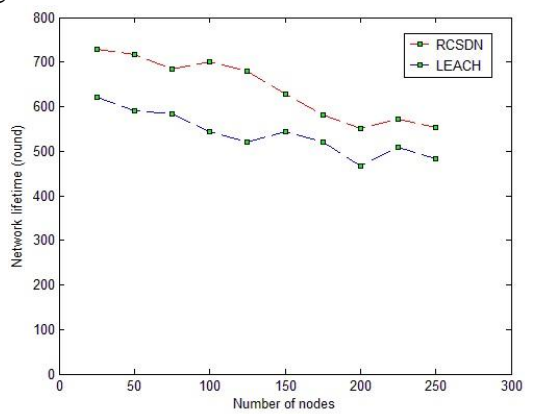

Fig. 3. Network lifetime with different number of nodes

\section{CONCLUSION}

In this paper we have presented a new algorithm to form clusters in sensor networks. The formation of optimal clusters plays an important role in increasing the lifetime and reliability of the network. With multiple simulations we have shown that the RCSDN can distribute clusters in an almost balanced manner in the network. We have demonstrated the presented algorithm in an efficient fashion and have compared it with the LEACH method in cluster formation; in which the results show a higher efficiency level of the RCSDN in node energy reduction and cluster distribution.

\section{REFERENCES}

[1] W. Jeong and S. Y. Nof, "Performance evaluation of wireless sensor network protocols for industrial applications," Journal of Intelligent Manufacturing, vol. 19, no. 3, 2008, pp. 335-345

[2] K. Sohrabi et al., "Protocols for self-organization of a wireless sensor network," IEEE Personal Communications, vol. 7, no. 5, 2000, pp. 16-27.

[3] R. Min, et al., "Low power wireless sensor networks," in Proceedings of International Conference on VLSI Design, Bangalore, India, January 2001.

[4] J. M. Rabaey et al, "PicoRadio supports ad hoc ultra low power wireless networking," IEEE Computer, vol. 33, no. 7, 2000, pp. 42-48.

[5] I. F. Akyildiz et al, "Wireless sensor networks: a survey," Computer Networks, vol. 38, no. 4, 2002, pp. 393-422.

[6] K. Akkays and M. Younis, "A Survey on Routing Protocols for Wireless Sensor Networks," Elsevier Ad Hoc Network Journal, vol. 3 , no. 3, May, 2005, pp. 325-349.

[7] W. R. Heinzelman, A. Chandrakasan, and H. Balakrishnan, "Energy efficient communication protocol for wireless sensor networks," in Proceedings of the $33^{\text {rd }}$ Hawaii International Conference on System Science, vol. 2, 2000.

[8] A. A. Abbasi and M. Younis, "A survey on clustering algorithms for wireless sensor networks," Computer Communications, vol. 30, 2007, pp. 2826-2841

[9] W. B. Heinzelman, A. Chandrakasan, and H. Balakrishnan, “An application-specific protocol architecture for wireless microsensor networks," IEEE Trans. Wireless Commun, vol. 1, no. 4, 2002, pp. $660-670$.

[10] O. Younis and S. Fahmy, Heed: "a hybrid, energy-efficient, distributed clustering approach for ad hoc sensor networks," IEEE Trans. Mobile Comput, vol. 23, no. 4, 2004, pp. 366-379.

[11] Q. Wang and W. Yang, "Energy consumption model for power management in wireless sensor networks," 4th Annual IEEE communications society conference on sensor, mesh and ad hoc communications and network (SECON 2007)

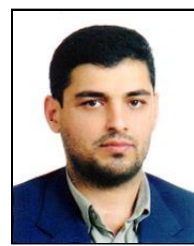

Arash Ghorbannia Delavar is the Director of Virtual University and Multimedia Training Department of Payame Noor University in IRAN.

$\mathrm{He}$ is also currently editor of many computer science journals in IRAN. His research interests are in the areas of computer networks, microprocessors, data mining, Information Technology, and E-Learning.

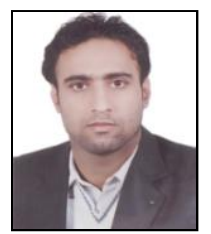

Javad Artin received the MS, in 2011 and now, he is the teacher in the department of Computer in Payame Noor University, Gorgan, IRAN. His research interests include computer networks, wireless communication, Genetic algorithm, and Fuzzy logic.

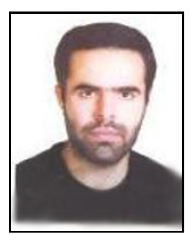

Mohammad Mahdi Tajari received the BS , in 2007 and Now, he is a Student the MS degree in the department of computer science in Islamic Azad University Mashhad, Iran. His research interests include wireless communication, network computer and Fuzzy logic. 